\title{
MIÍASE BUCAL - REVISÃO DA LITERATURA
}

\author{
ORAL MYIASIS - A REVIEW OF LITERATURE \\ Jaisson Cencil ${ }^{1}$, Maurício Zardo ${ }^{1}$, André Takahashi ${ }^{2}$, \\ Antonio Carlos Domingues de Sá ${ }^{3}$, Luciana Dorochenko Martins ${ }^{3}$, \\ Ramon César Godoy Gonçalves ${ }^{3}$ \\ 1 Universidade Estadual de Ponta Grossa - UEPG, Departamento de Odontologia, Campus \\ em Uvaranas, Ponta Grossa, PR \\ 2 Autor para contato: Universidade Católica - PUC, RS, Brasil; (51) 9997-6100; \\ e-mail:drwstk@usp.br \\ 3 Associação Brasileira de Odontologia - Secção Regional de Ponta Grossa/PR - ABO, \\ Curso de Especialização em Cirurgia e Traumatologia Buco-maxilo-faciais, \\ Ponta Grossa, PR \\ Recebido para publicação em 02/03/2006 \\ Aceito para publicação em 02/06/2006
}

\section{RESUMO}

A miíase bucal é a infestação dos tecidos bucais por larvas de moscas. Ocorre principalmente em países tropicais associados com condições de saneamento básico e higiene deficitárias. Esse artigo faz uma revisão dos relatos de casos e dos tratamentos para miíase bucal.

Palavras-chave: Miíase bucal, cavidade bucal, ivermectina

\begin{abstract}
Oral myiasis is the invasion of oral tissues by larvae of flies. It occurs mainly in the tropic, and associated with inadequate public and personal hygiene. This es a review of cases reports and of treatments for oral myíasis.
\end{abstract}

Key words: Oral myiasis; oral cavity; ivermectin

\section{Introdução}

Miíase é a invasão de tecidos vivos ou órgãos por larvas de mosca (Aguiar, Enwonwu, Pires 2003;
Anil, Jacob, Hari 1989). Essa infestação pode ser cutânea, subcutânea, em feridas, cavitária facial (nariz, pálpebra, seios da face, ouvido, boca), gastrointestinal, vaginal ou generalizada. É classificada como: 
a) Obrigatórias (primárias ou biontófagas), causadas por larvas de dípteros, que naturalmente se desenvolvem sobre ou dentro de vertebrados, alimentando-se de tecidos vivos; b) Facultativas (secundárias ou necrobiontófogas), causadas por larvas de dípteros que usualmente se desenvolvem em matéria orgânica em decomposição, mas eventualmente podem atingir tecidos necrosados em um hospedeiro vivo; c) Pseudomiíase (acidental), causadas por larvas de dípteros ingeridas com alimentos, podendo ocasionar distúrbios mais ou menos graves , tais como infecções intestinais (Linhares 2000; Rey 2002).

As miíases são mais comuns em países do terceiro mundo, como os da América Latina, África, Oriente Médio e Ásia, mas podem ocorrer em países desenvolvidos ou em turistas em viagem a países tropicais. A história e características dos pacientes podem incluir: visita a áreas endêmicas, má higiene, senilidade, crianças (Al-Ismaily, Scully 1995; Bhatt, Jayakrishnan 2000; Erol, et al. 2000), pacientes portadores de doenças mentais, hemiplegia, epiléticos, morador de áreas rurais, presença de tecidos necrosados, etilismo, desnutrição, falta de selamento labial, resistência corporal diminuída, respiração bucal, mordida aberta anterior, doença periodontal (Gunbay, et al. 1995; Gursel, et al. 1995), infecção dental crônica, halitose severa, extração dental (Bozzo et al. 1992), traumatismos faciais (Baliga et al. 2001; Novelli, Haddock, Eveson 1993), lesões necróticas (Noma) (Aguiar, Enwonwu, Pires 2003).

Mais de 150 espécies de dípteros podem causar miíase em humanos. Várias espécies têm sido relacionadas à miíase bucal (Al-Ismaily, Scully 1995; Bozzo et a. 1992; Erol et al. 2000; Ng et al. 2003). No Brasil, a mosca Cochliomyia hominivorax (Townsend, Calliphoridae) (Figura 1), que ocorre principalmente em áreas tropicais, é a espécie de mosca mais importante como produtora de miíase bucal (Duque, Valderrama, Gonzalez 1998; Gomez et al. 2003; Shinohara et al. 2004). Suas larvas causam a mí́ase conhecida popularmente como bicheira (Ribeiro et al. 2001).

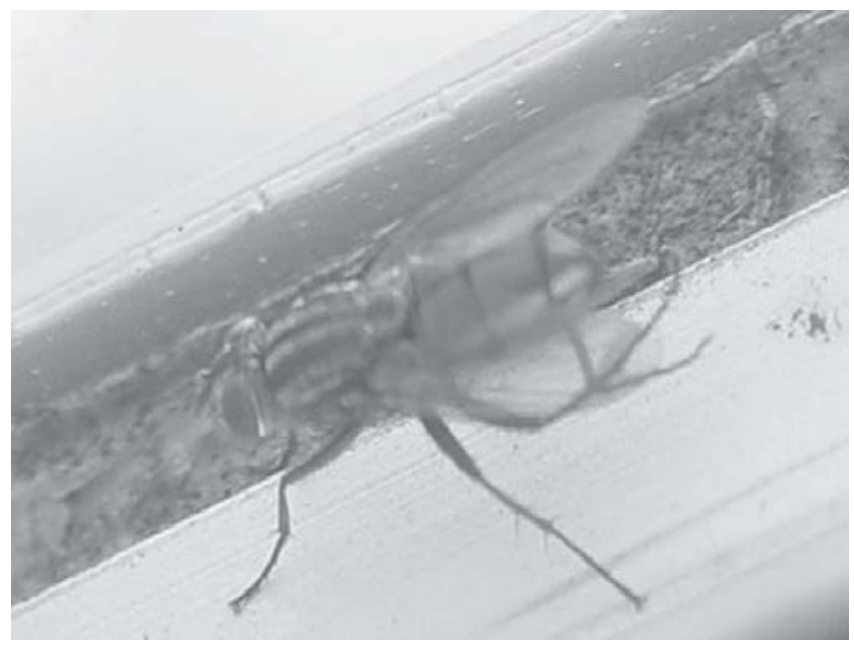

Figura 1 - Cochliomyia hominivorax - Mosca varejeira

\section{A MOSCA : Cochliomyia hominivorax}

Conhecida popularmente como mosca varejeira, é a mais importante espécie causadora de miíase primária, distribuindo-se desde o sul dos EUA até o norte do Chile e Argentina. O adulto mede cerca de 8 mm de comprimento, possui corpo de coloração verde, com reflexos azul-metálico em todo o tórax e abdômen. A larva madura (Figura 2), de coloração brancoamarelada, mede aproximadamente $15 \mathrm{~mm}$ no comprimento. Apresenta dois estigmas respiratórios na extremidade posterior, cada um com três espiráculos mais próximos na base; as traquéias aparecem bem pigmentadas até o terceiro ou quarto segmento larvar. 


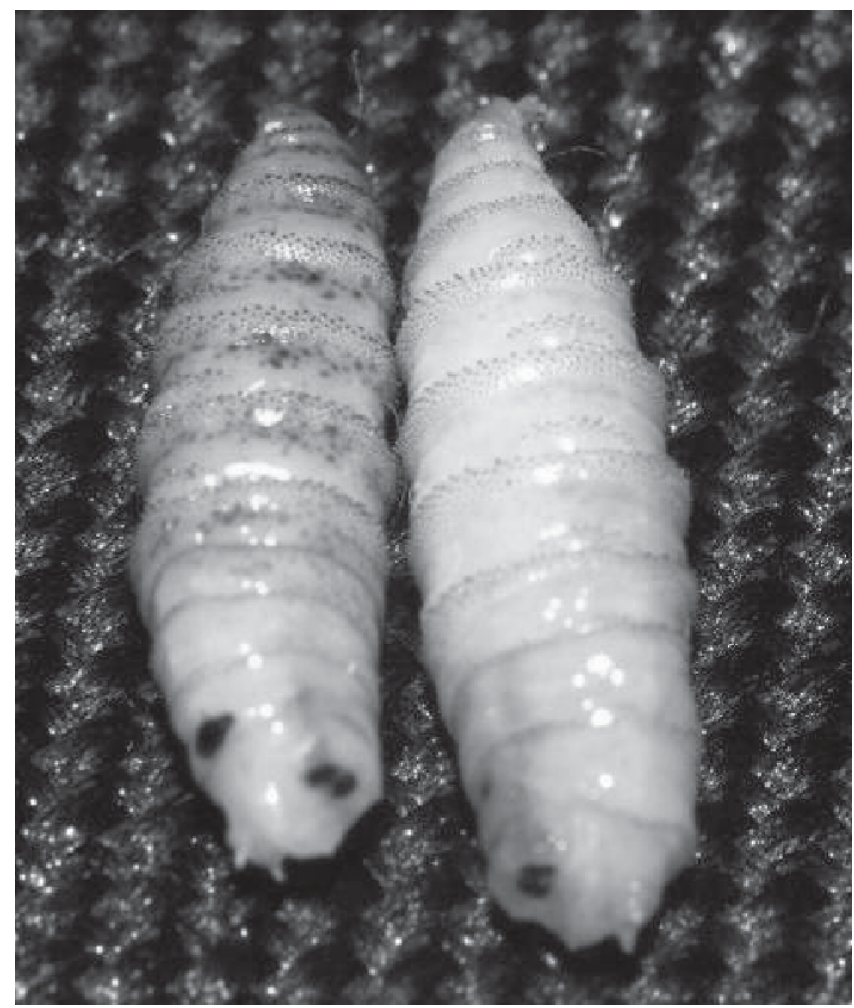

Figura 2 - Larvas maduras de Cochliomyia hominivorax

As moscas adultas são excelentes voadoras, percorrendo distância de até $17 \mathrm{~km}$ em um dia (Linhares 2000). São mais abundantes nos climas quente eúmido, notadamente nos meses chuvosos de verão. Os adultos copulam uma única vez, cerca de cinco dias depois de emergirem. Logo após a cópula, iniciam a postura nas cavidades naturais do corpo de seu hospedeiro, como narinas, canal auditivo, vulva, ou em feridas recentes, incisões cirúrgicas, etc. As fêmeas depositam de 10 a 200 ovos nas cavidades ou às margens de feridas e arranhões. Põem ovos a cada quatro dias, num total de até 2800 ovos ao longo de sua vida (aproximadamente 65 dias, em condições de laboratório). Em menos de 24 horas, as larvas, conhecidas vulgarmente como "bicheiras", eclodem e começam a alimentar-se vorazmente de tecidos vivos (larvas biontófagas) do hospedeiro. Essas larvas produzem enzimas proteolíticas que digerem os tecidos formando lesões extensas. O parasitismo dura de quatro a oito dias, quando as larvas maduras saem espontaneamente do hospedeiro, caem no solo, enterram-se e encasulam. Cerca de oito dias após, emergem como adultos. Estas moscas alimentam-se de néctar, suco de frutas e secreções orgânicas. O ciclo evolutivo desta espécie dura aproximadamente 25 dias, dependendo das condições climáticas (Linhares 2000; Rey 2002).

\section{Diagnóstico}

As manifestações clínicas da miíase não são específicas e variam de acordo com a área do corpo envolvida e com a espécie de mosca. Sinais e sintomas gerais podem incluir febre, mialgia, artralgia, hipereosinofilia, taxa de sedimentação de eritrócitos elevada e reação inflamatória no local da infestação, prurido, dor, tumefação e mobilidade do local. Na boca o local de prevalência é a área dos dentes superiores anteriores. $\mathrm{O}$ aspecto pode variar de pequenas úlceras a grandes destruições de tecido com a presença de larvas (Figura 3). A miíase deve ser diferenciada de lesões como gengivo estomatite inflamatória, inflamação papilar, lesões exofitica inflamatórias (ex granuloma piogênico), abscesso periodontal e manifestações bucais de câncer (ex leucemia). A constatação da presença de larvas no tecido é a chave para o diagnóstico da miíase, entretanto vale salientar que ela pode estar associada a lesões tumorais ou necróticas. A visualização das larvas no tecido é a chave nesse diagnóstico. Exames imaginológicos auxiliam na avaliação da destruição tecidual e extensão da infestação.

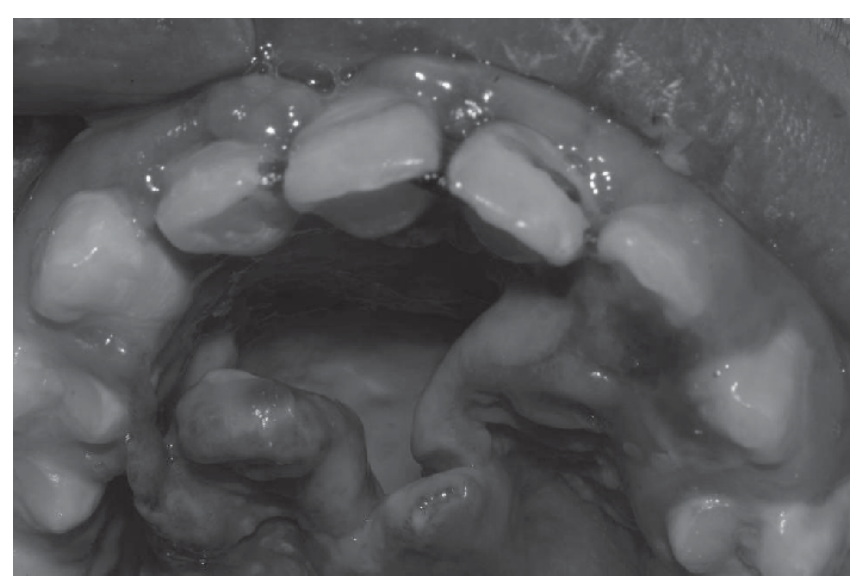

Figura 3 - Grande destruição tecidual causada pelas larvas 


\section{Tratamento}

O tratamento baseia-se na remoção das larvas e dos tecidos necrosados (Felices, Ogbureke 1996; Henry 1996; Hopkins 2003; Lata , Kapila, Aggarwal 1996; Zeltser, Lustmann 1988). A quantidade de larvas a serem removidas deve ser considerada na indicação do tipo de tratamento.

Quando a miíase for por poucas larvas e a fuga delas para cavidades ou profundidade de tecidos estiver limitada, o tratamento é apenas mecânico, com a remoção individual das larvas. Esta remoção é um processo doloroso, incômodo e constrangedor, quer para o paciente quer para o profissional. A literatura ressalta o emprego de inúmeros medicamentos tópicos asfixiantes como o iodofórmio em pó para forçar a saída das larvas. O uso desses medicamentos pode torná-las agitadas e migrarem para regiões ainda mais inabordáveis. Medicamentos sistêmicos como o óleo canforado, o oxicianureto de mercúrio, o sulfureto de mercúrio, entre outros, foram tentados, sempre no sentido de expulsar as larvas, mas com resultados pouco eficazes (Linhares 2000).

Quando houver muitas larvas o tratamento com uma dose de ivermectina $6 \mathrm{mg}$ é recomendado ( Shinohara et al. 2004; Campbell 1985; De Tarso et al. 2004; Martin-Prevel et al. 1993; Shinohara 2003). A ivermectina pertence ao grupo químico das avermectinas, um antibiótico macrolídeo semi-sintético, isolado do Streptomyces avermitilis sendo que sua ação consiste em interferir na transmissão de impulsos nervosos, junto às terminações nervosas, por estímulo à liberação do ácido gama amino butírico (GABA). Em vermes redondos (nematódeos), a ivermectina estimula a liberação do GABA nas terminações nervosas, favorecendo sua fixação nos receptores, interrompendo dessa forma os impulsos nervosos, determinando a eliminação dos parasitas. A acetilcolina, principal neurotransmissor periférico em mamíferos, não é afetada pela ivermectina. A ivermectina não penetra com facilidade através da barreira do sistema nervoso central de mamíferos, onde o GABA atua como neurotransmissor.

A remoção dos tecidos inviáveis (Figura 4) e o exame anatomopatológico são feitos para excluir necrose tecidual prévia à infestação (Aguiar, Enwonwu, Pires 2003).

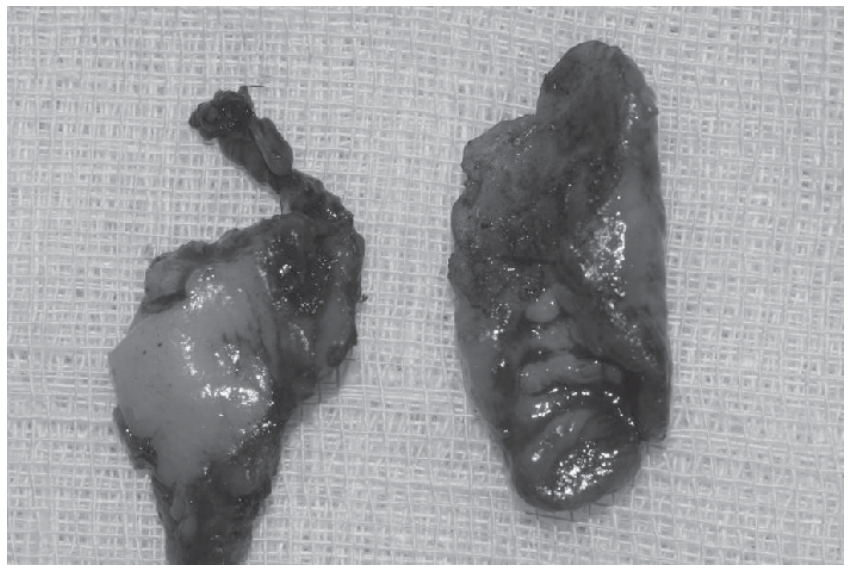

Figura 4 - Remoção do tecido para análise histológica

As larvas devem ser levadas para identificação entomológica, embora o diagnóstico exato da espécie de mosca responsável pela infestação não possa ser feito com base no estágio de larva. As larvas obtidas dos pacientes devem ser guardadas na carne ou em um meio sintético até seu estágio de pulpa e eventualmente até emergir como mosca adulta, nesse tempo é possível determinar a espécie baseado nas características morfológicas.

Além de recomendações de higiene, a erradicação de moscas no ambiente cotidiano do paciente deve ser considerada como extensão do tratamento, principalmente na miíase primária. A bicheira foi erradicada dos Estados Unidos após um programa implantado pelo governo americano, em que foram integrados o controle químico com inseticidas organofosforados e a técnica de liberação de machos esterilizados com radiação gama (Linhares 2000).

\section{Considerações finais}

A miíase bucal por Cochliomyia hominivorax acontece no Brasil, causando uma série enorme de prejuízos aos criadores de animais. Embora o hospedeiro preferencial seja o gado, ocasionalmente a postura de ovos pode ocorrer nos seres humanos, notadamente aqueles debilitados ou portadores de deficiências mentais. Na cavidade bucal, não raro, observa-se extensa destruição dos tecidos, com aspecto desagradável. O tratamento com dose única 
de ivermectina é de custo reduzido e leva à imediata destruição das larvas o que deve ser seguido pelo debridamento da ferida cirúrgica.

\section{REFERÊNCIAS}

1. AGUIARAM, ENWONWU CO, PIRES FR. Noma (cancrum oris) associated with oral myiasis in an adult. Oral Dis. 2003 May;9(3):158-9.

2. Al-ISMAILY M, SCULLY C. Oral myiasis: report of two cases. Int J Paediatr Dent. 1995 Sep;5(3):177-9.

3. ANIL S, JACOB OA, HARI S. Oral myiasis: a case report. Ann Dent. 1989 Winter;48(2):28-30.

4. BALIGA MJ, DAVIS P, RAI P, RAJASEKHAR V. Orbital myiasis: a case report. Int J Oral Maxillofac Surg. 2001 Feb;30(1):83-4.

5. BHATT AP, JAYAKRISHNAN A. Oral myiasis: a case report. Int J Paediatr Dent. 2000 Mar;10(1):67-70.

6. BHOYAR SC, MISHRAYC. Oral myiasis caused by diptera in epileptic patient. J Indian Dent Assoc. 1986 Dec;58(12):5357. BOZZO L, LIMA IA, DE ALMEIDA OP, SCULLY C. Oral myiasis caused by sarcophagidae in an extraction wound. Oral Surg Oral Med Oral Pathol. 1992 Dec;74(6):733-5.

8. CAMPBELL WC. Ivermectin: An update. Parasitol Today 1985;1:10-16.

9. DE TARSO P, PIERRE-FILHO P, MINGUINI N, PIERRE LM, PIERRE AM. Use of ivermectin in the treatment of orbital myiasis caused by Cochliomyia hominivorax. Scand J Infect Dis. 2004;36(6-7):503-5.

10. DUQUE SFL, VALDERRAMA HR, GONZALEZ RJ. Tratamiento de miasis oral com ivermectina. Notificación de três casos causados por Cochliomyia hominivorax (Coquerel). Rev Fac Odontol Univ Ant 1998;10:41-47.

11. EROLIB, UNLUG BALCI K, TANRIKULU R. Oral myiasis caused by hypoderma bovis larvae in a child: a case report. J Oral Sci. 2000 Dec;42(4):247-9.

12. FELICES RR, OGBUREKE KU. Oral myiasis: report of case and review of management. J Oral Maxillofac Surg. 1996 Feb;54(2):219-20.

13. GOMEZ RS, PERDIGÃO PF, PIMENTA FJ, RIOS LEITE
Leite AC, TANOS DE LACERDA JC,CUSTÓDIO NETO AL. Oral myiasis by screwworm Cochliomyia hominivorax. $\mathrm{Br} \mathrm{J}$ Oral Maxillofac Surg. 2003 Apr;41(2):115-6.

14. GUNBAY S, BICAKCI N, CANDAT, CANDA S. Acase of myiasis gingiva. J Periodontol. 1995 Oct;66(10):892-5.

15. GURSEL M, ALDEMIR OS, OZGUR Z, ATAOGLU T.A rare case of gingival myiasis caused by diptera (Calliphoridae). J Clin Periodontol. 2002 Aug;29(8):777-80.

16. HENRY J. Oral myiasis: a case study. Dent Update. 1996 Nov;23(9):372-3.

17. HOPKINSs R. Oral myiasis. Br J Oral Maxillofac Surg. 2003 Dec;41(6):422.

18. LATA J, KAPILA BK, AGGANWAL P. Oral myiasis. A case report. Int J Oral Maxillofac Surg. 1996 Dec;25(6):455-6.

19. LINHARES AX. Miíases. In: NEVES, D.P.; MELO, A.L.; GENARO, O. \& LINARDI, P.M. Parasitologia Humana. $10^{\mathrm{a}}$ ed. São Paulo: Ed. Atheneu, 2000. Cap. 48, p. 350-57.

20. MARTIN-PREVEL Y, COSNEFROY JI, TSHIPAMPA P, NGARIi P, CHODAKEWITZ JÁ, PINDER M. Tolerance and efficacy of single high-dose ivermectin for the treatment of loiasis. Am J Trop Med Hyg 1993;48:186-192.

21. NG KH, YIP KT, CHOI CH, YEUNG KH, AUYEUNG TW, TSANG AC, CHOW L, QUE TL. A case of oral myiasis due to Chrysomya bezziana. Hong Kong Med J. 2003 Dec;9(6):4546.

22. NOVELLI MR, HADDOCK A, EVESON JW. Orofacial myiasis. Br J Oral Maxillofac Surg. 1993 Feb;31(1):36-7.

23. REV, L. Bases da Parasitologia Humana. $2^{\text {a }}$ ed. Rio de Janeiro: Guanabara Koogan, 2002.

24. RIBEIROFAQ, PEREIRACSB, ALVESA, MARCON MA. Tratamento da miíase humana cavitária com ivermectina oral. Ver Brás Otorrinolaringol 2001;67:755-761.

25. SHINOHARA EH, MARTINI MZ, OLIVEIRA Neto HG, TAKAHASHI A. Oral myiasis treated with ivermectin: Case report. Braz Dent J 2004;15(1):79-81.

26. SHINOHARA EH. Treatment of oral myiasis with ivermectin. Br J Oral Maxillofac Surg. 2003 Dec;41(6):421.

27. ZELTSER R, LUSTMAN J. Oral myiasis [corrected and issued with original paging in Int J Oral Maxillofac Surg 1988 Oct;17(5):288-9] Int J Oral Maxillofac Surg. 1989 Apr;18(2):2889. 